\title{
One step route to ultra-long Ag nanowires for solar cells
}

\author{
Weiwei He ${ }^{1,2, a}$, Xiaohu Yan ${ }^{1}$, Yifei Long ${ }^{1}$, Chan Pan ${ }^{1}$, Junli Zhao ${ }^{1}$, Yuming \\ liang ${ }^{1}$ and Qiuxin Liu ${ }^{3, b}$ \\ ${ }^{1}$ City College, Wuhan University of Science and Technology, Wuhan 430083, China. \\ ${ }^{2}$ State Key Laboratory of Materials Processing and Die \& Mould Technology, Huazhong University \\ of Science and Technology, Wuhan 430074, China. \\ ${ }^{3}$ Wuhan University of Science and Technology, Wuhan, 430080, China. \\ aheww1987@126.com, ${ }^{\mathrm{b}}$ liuqiuxin1956@sohu.com
}

Keywords: Ag nanowire; ultra-long, transparent electrode.

Abstract:Currently, the most commonly used TCFs in the electronics industry is indium tin oxide (ITO). However, ITO has some inherent shortcomings, such as high cost and brittle nature, which limited its widespread application. Recent studies have investigated alternatives to ITO. Ag nanowires (AgNWs) is still one of the best material for electronics. This paper reports a method for ultra-long AgNWs with just one step route and apply them as an electrode to Si/PEDOT: PSS solar cells. Through the only one step route, we could dramatically increase the length of the AgNWs form $10 \mu \mathrm{m}$ to even over $100 \mu \mathrm{m}$ with the development of a novel intermittent growth method. By intermittently adding $\mathrm{Ag}^{+}$solution, the length of nanowires could be controlled in the range of 20-100 $\mu \mathrm{m}$ while diameter of $\sim 100 \mathrm{~nm}$ with narrow size dispersion was maintained. In addition, Si nanoarray (SiNA)/PEDOT: PSS hybrid solar cells are fabricated with AgNWs electrodes. And the efficiency of solar cells with ultra-long AgNWs electrodes can reach 6\% which is $40 \%$ higher than that of ITO electrodes. The ultra-long AgNWs can be applied for various opto-electronics and ultimately for future wearable electronics.

\section{Introduction}

Currently, the most commonly used TCFs in the electronics industry is indium tin oxide (ITO) [1-3]. Its excellent optical transparency and low sheet resistance have extended their use as electrodes in highly demanding applications [4-5]. And Indium tin oxide (ITO) is the most wildly used TE that account for $93 \%$ of the market. However, there are many problems which limit its further development.[6]

Recent studies have investigated alternatives to ITO such as carbon nanotubes (CNT) [7-10], graphene [11-13] and metallic nanostructures [14-16]. It seems that the closer approach to ITO, the more likely to be recognized as a substitute. Fortunately, metallic nanostructures have attracted much attention for a decade due to their unique optoelectronic properties and potential applications in TCFs. A variety of metallic nanostructures such as copper [17-19], gold [20-22], silver [23-25] nanowires have been investigated as promising candidates for replacing ITO.

Recently, silver nanowires (AgNWs) have been widely studied in fabricating transparent conductive materials and devices because of their high conductivity, good flexibility and mature preparation technology. They play very important roles in practical devices such as displays, touch panels, capacitors, batteries, transparent and flexible electrodes and water filters. Organic light emitting diodes (OLEDs), touch panels, and solar cells.

Many ways to synthesize AgNWs have been reported. In 2002, Xia et al. demonstrated a solution-phase method that generates silver nanowires by reducing silver nitrate with ethylene glycol in the presence of PVP at $160^{\circ} \mathrm{C}$. This polyol-process method can overcome the shortcomings that the old template-based methods had. Since then, many efforts have been made to enhance the properties of AgNWs. Lee et al. presented a successive multistep growth (SMG) method that very long AgNWs can be synthesized at $151.5^{\circ} \mathrm{C}$. Very long NWs were synthesized by 7 steps of SMG process, however, the long-time process may produce extra time and money cost. 
In this paper, we developed a novel one-step growth method to synthesize very long AgNWs at a very low temperature of $130^{\circ} \mathrm{C}$. Through the only one step route, we could dramatically increase the length of the AgNWs form $10 \mu \mathrm{m}$ to even over $100 \mu \mathrm{m}$ with the development of a novel intermittent growth method. By intermittently adding $\mathrm{Ag}^{+}$solution, the length of nanowires could be controlled in the range of $10-100 \mu \mathrm{m}$ while diameter of $\sim 100 \mathrm{~nm}$ with narrow size dispersion was maintained. In addition, Si nanoarray (SiNA)/PEDOT: PSS hybrid solar cells are fabricated with AgNWs electrodes. And the efficiency of solar cells with AgNWs electrodes can reach 6\% which is $40 \%$ higher than that of ITO electrodes. The ultra-long AgNWs can be applied for various opto-electronics and ultimately for future wearable electronics.

\section{Experiment}

Synthesis of ultra-long Ag nanowires by one step route. Ethylene glycol (EG) solutions of $\mathrm{Ag}^{+}$and $\mathrm{Cl}^{-}$were separately prepared by dissolving powders of silver nitrate (along with polyvinylpyrrolidone (PVP)) and ferric chloride into two EG solutions. For $\mathrm{Ag}^{+}$solution, $30 \mathrm{~mL}$ EG solution of 0.052 $\mathrm{mol} / \mathrm{L} \mathrm{AgNO}_{3}$ and $0.067 \mathrm{~mol} / \mathrm{L} \mathrm{PVP}$ was thoroughly agitated. For $\mathrm{Cl}^{-}$solution, room temperature EG solution of $6 \times 10^{-4} \mathrm{~mol} / \mathrm{L} \mathrm{FeCl}_{3}$ was first prepared and then $2.5 \mathrm{~mL}$ solution was extracted and dropped into $30 \mathrm{~mL} E G$ which was previously heated at $130^{\circ} \mathrm{C}$ for $1 \mathrm{~h}$. After further heating and agitating of $\mathrm{Cl}^{-}$solution for 5-10 min, $\mathrm{Ag}^{+}$solution was added with a dropping speed of $0.5 \mathrm{ml} / \mathrm{min}$. Twenty minutes (10 $\mathrm{mL} \mathrm{Ag}^{+}$solution dropped) later, dropping was stopped and the mixed precursor solution was agitated and heated for $10 \mathrm{~min}$. This adding and precursor reaction process was repeated for 2 times. Finally, post-reaction solution was cooled naturally and then filtered by qualitative filter paper (intermediate speed). AgNWs were washed out of the filter paper with deionized water. After separation by centrifugation at speed of $2000 \mathrm{rpm}$, deposit of Ag nanowires was washed by repeating the ultra-sonication in ethanol and centrifugation process.

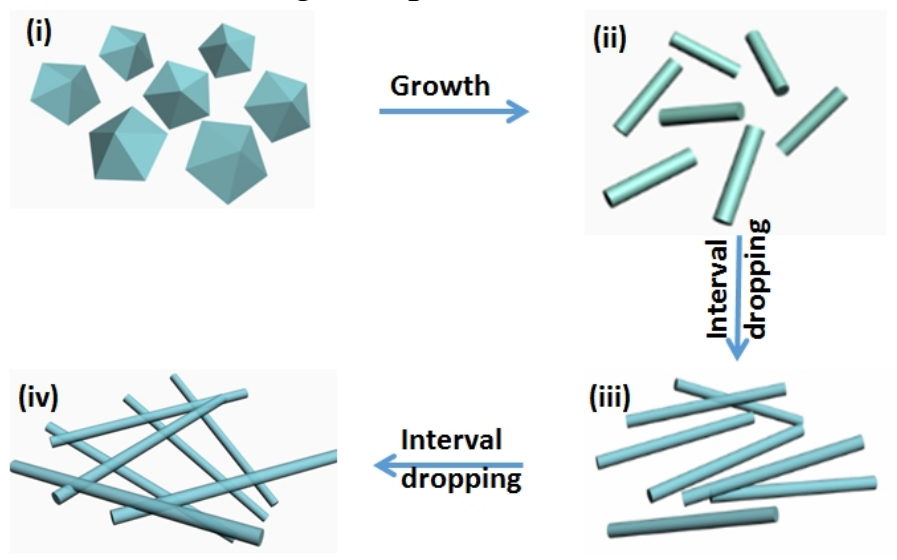

Figure 1. Flow-process diagram of synthesis of ultra-long Ag nanowires by one step route.

Characterizations. The morphologies of ultra-long Ag nanowires and films were characterized by optical microscopy (OM) (Olympus BX51, Microscopes Inc.), scanning electron microscopy (SEM) (Sirion 200 FEG) and transmission electron microscopy (TEM) (JEM-2010 transmission electron microscope equipped with an Oxford INCA energy dispersive spectrometer (EDS)).

\section{Results and discussions}

Successful flexible and transparent conductors need to achieve superior optical transparency, electrical conductivity and mechanical flexibility simultaneously. The length and the diameter of the Ag NWs play very important roles in conductive metal NW network electrodes for flexible transparent conductor. However, the most of metal NWs synthesized by wet chemistry were limited to 1-20 $\mu \mathrm{m}$ in length and showed limited transparency, electrical conductivity and mechanical robustness. Through the development of a novel intermittent growth method, we could dramatically 
increase the length of the AgNWs even over $100 \mu \mathrm{m}$ with only one step route (Fig. 2). Figure 2 shows the growth process of extra long silver nanowires. First of all, silver particles are generated (Fig. 2a). Twenty minutes (10 $\mathrm{mL} \mathrm{Ag}^{+}$solution dropped) later, dropping was stopped and the mixed precursor solution was agitated and heated for $10 \mathrm{~min}$. At this time, the length of AgNWs is about $2 \mu \mathrm{m}$ (Fig. 2a). After this adding and precursor reaction process was repeated for 2 times, AgNWs can grow to even over $100 \mu \mathrm{m}$. This may be because AgNWs to grow along the original nanowire when we add silver again.

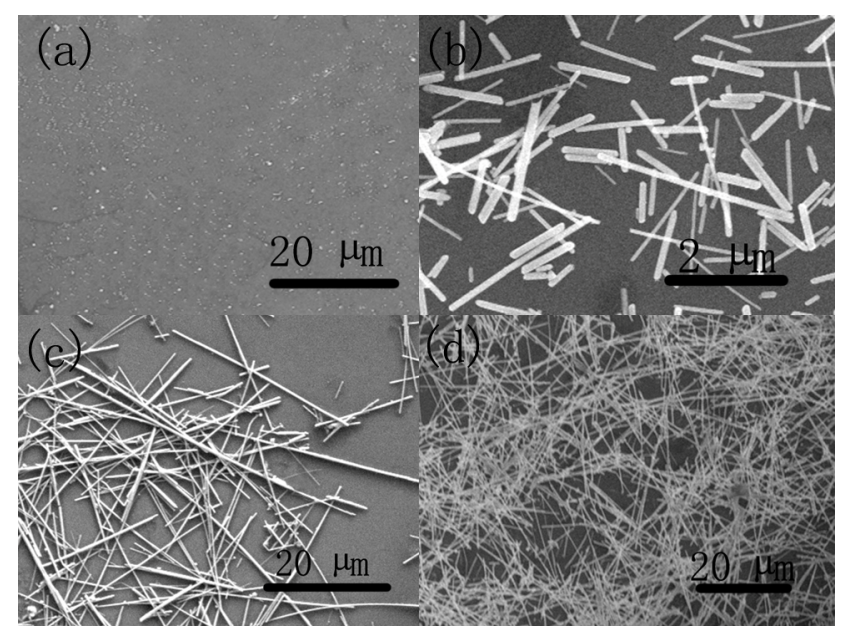

Figure 2. The growth process of extra long silver nanowires.

Figure 3 is the AgNW length distribution graph and corresponding SEM images of original Ag NWs (top graph and inset picture) and ultra-long Ag NWs by one step route process (bottom graph and inset picture). As shown in Figure 3, the length of nanowires could be controlled in the range of 20-100 $\mu \mathrm{m}$ while diameter of $\sim 100 \mathrm{~nm}$ with narrow size dispersion was maintained by intermittently adding $\mathrm{Ag}^{+}$solution,. Figure 1a shows that the average length of initially grown AgNWs was $10.2 \mu \mathrm{m}$ (4.6 $\mu \mathrm{m}$ standard deviation) and one step route grown AgNWs was $95.1 \mu \mathrm{m}$ (46.7 $\mu \mathrm{m}$ standard deviation). Aspect ratio as high as 1000 of these ultra-long nanowires benefits transparence of those Ag nanowire films whose resistance are sufficiently low.

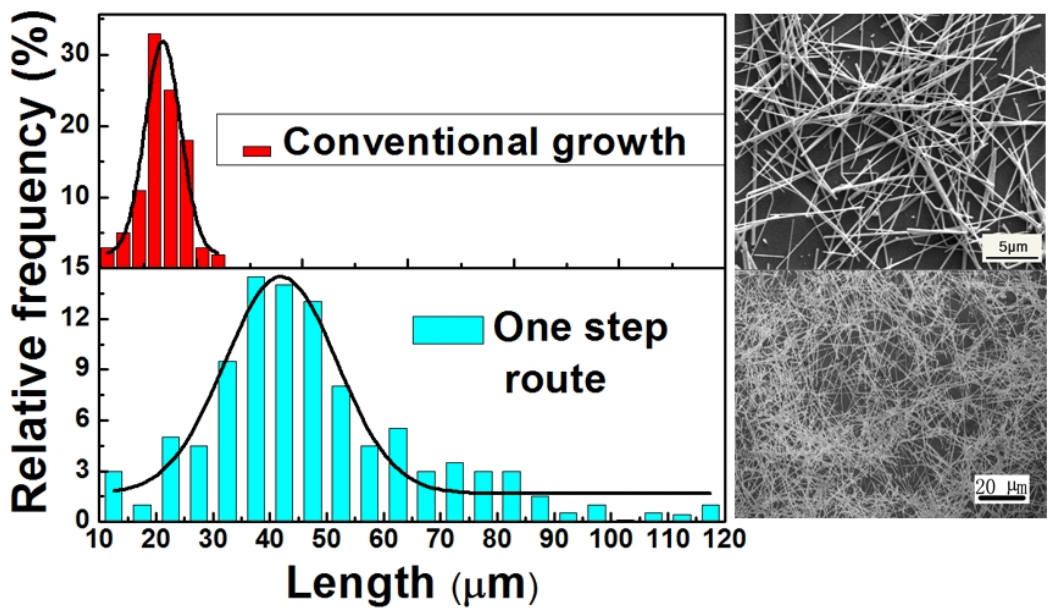

Figure 3. The AgNWs length distribution graph and corresponding SEM images of original Ag NWs (top graph and inset picture) and ultra-long Ag NWs by one step route process (bottom graph and inset picture).

Ag nanowires having structure of face centered cubic (fcc) grow preferentially in $\langle 110\rangle$ direction (Fig. 4). A twin plane of $\{111\}$ faces has also been observed in TEM image which was reported before. Two sets of spots in diffraction pattern support the fact and interplanar spacings agree with those from HRTEM image. No obvious impurities such as surface oxides were detected for fresh Ag nanowires according to TEM EDS spectrum. Interestingly, we found that some Ag nanowires are curving with a 
corner of different angles. It is probably due to bizarre growth mechanism which deserves in-depth study.

Highly crystallized Ag nanowires were synthesized by the one step route. By intermittently adding $\mathrm{Ag}^{+}$solution, the length of nanowires could be controlled in the range of 10-100 $\mu \mathrm{m}$ while diameter of $\sim 100 \mathrm{~nm}$ with narrow size dispersion was maintained. Aspect ratio as high as 1000 of these ultra-long nanowires benefits transparence of those $\mathrm{Ag}$ nanowire films whose resistance are sufficiently low. Ag nanowires having structure of face centered cubic (fcc) grow preferentially in $\langle 110\rangle$ direction. A twin plane of $\{111\}$ faces has also been observed in TEM image which was reported before. Two sets of spots in diffraction pattern support the fact and interplanar spacings agree with those from HRTEM image. No obvious impurities such as surface oxides were detected for fresh Ag nanowires according to TEM EDS spectrum. Interestingly, we found that some Ag nanowires are curving with a corner of different angles. It is probably due to bizarre growth mechanism which deserves in-depth study.

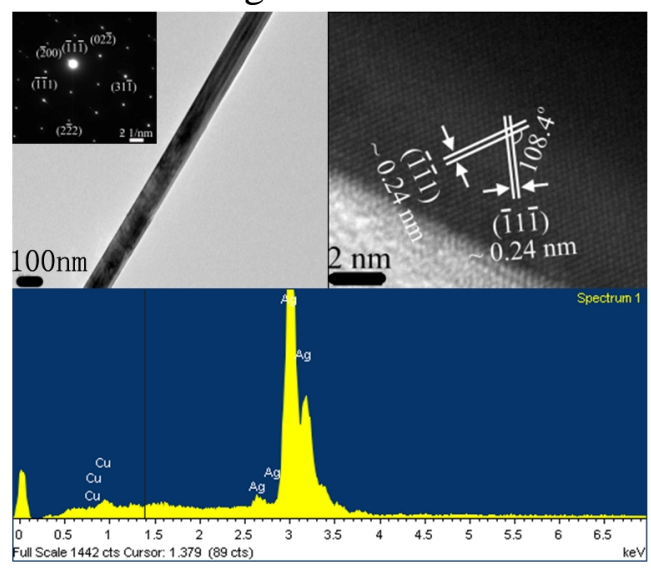

Figure 4. The TEM image of an individual silver nanowire. The inset gives the micro diffraction pattern recorded by focusing the electron beam on this wire (top left graph and inset picture). The High-resolution TEM image showing the structure of face centered cubic (fcc) (top right graph and inset picture). The TEM EDS spectrum of Ag NWS showing no obvious impurities (bottom graph).

Si nanoarray (SiNA)/PEDOT: PSS hybrid solar cells are separately fabricated with ultra-long AgNWs, ITO, and $10 \mu \mathrm{m} \mathrm{AgNWs} \mathrm{electrodes.} \mathrm{Fig.} \mathrm{5a} \mathrm{shows} \mathrm{the} \mathrm{diagram} \mathrm{of} \mathrm{the} \mathrm{solar} \mathrm{cells} \mathrm{with}$ AgNWs electrodes that replace the traditional ITO electrode. Fig $5 \mathrm{~b}$ shows the J-V curves of solar cell with different electrodes. The performance of the solar cells with ultra-long AgNWs electrodes is obviously better than the others. And the efficiency of solar cells with ultra-long AgNWs electrodes can reach 6\%, while the efficiency with ITO electrodes is 3.5\% and the efficiency with $10 \mu \mathrm{m} \mathrm{AgNWs}$ electrodes is $2 \%$. We can see that the efficiency of solar cells with ultra-long AgNWs electrodes is $40 \%$ higher than that of ITO electrodes, and 67\% higher than that of $10 \mu \mathrm{m} \mathrm{AgNWs} \mathrm{electrodes.} \mathrm{As}$ shown in Figure 5, solar cell with ultra-long AgNWs electrodes has the same short-circuit current with that of ITO electrodes. This means that both of the two electrodes can provide sufficient carrier transport rates. However, solar cell with ultra-long AgNWs electrodes has higher open circuit voltage. This may be because silver nanowire electrodes have stronger optical scattering for more photogenerated carriers. Moreover, we see that solar cell with $10 \mu \mathrm{m}$ AgNWs electrodes has the lowest efficiency. This mainly due to its low carrier transport properties. Consequently, solar cell with ultra-long AgNWs electrodes has the highest efficiency may due to the ultra-long AgNWs electrodes can provide better carrier transport properties as well as stronger light scattering. 

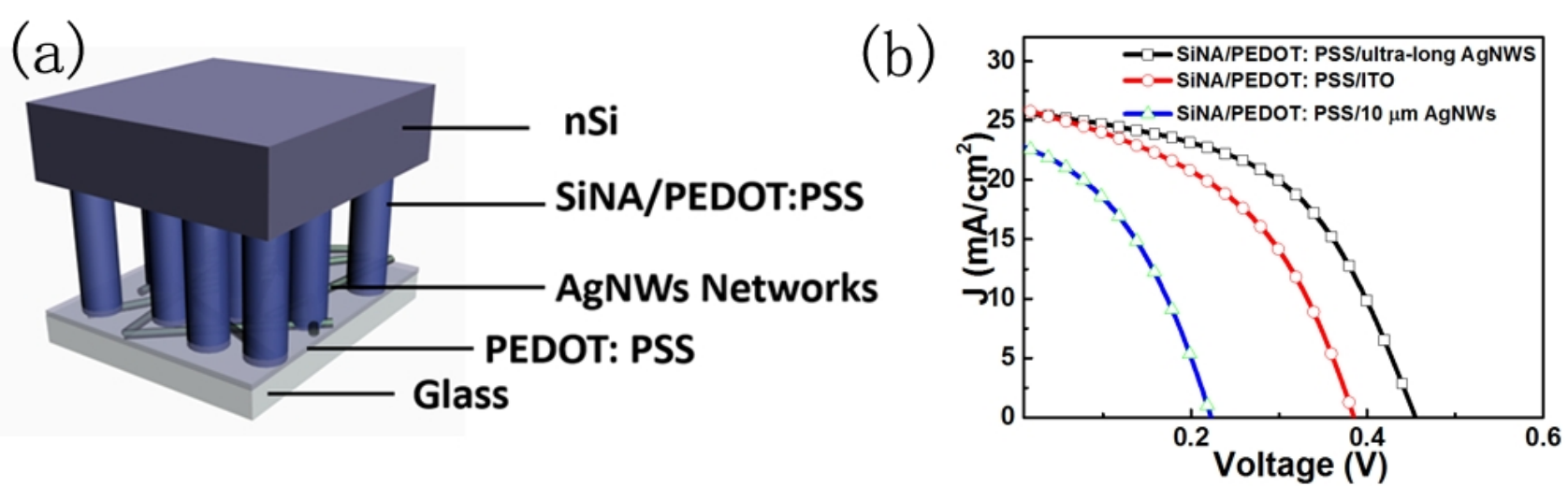

Figure 5. Diagram and J-V curves of SiNA/PEDOT: PSS hybrid solar cells. (a) Diagram of SiNA/PEDOT: PSS hybrid solar cells with $\mathrm{AgNWs} / \mathrm{SnO}_{2} \cdot \mathrm{xH}_{2} \mathrm{O}$ composite electrode. (b) J-V curves of solar cell with $\mathrm{AgNWs}$ and $\mathrm{AgNWs} / \mathrm{SnO}_{2} \cdot \mathrm{xH}_{2} \mathrm{O}$ composite electrodes.

\section{Conclusion}

Currently, the most commonly used TCFs in the electronics industry is indium tin oxide (ITO). However, ITO has some inherent shortcomings, such as high cost and brittle nature, which limited its widespread application. Obviously, the ultimate market opportunity for alternative TEs is in fully flexible devices. Recent studies have investigated alternatives to ITO. Ag nanowires (AgNWs) is still one of the best material for electronics. Successful flexible and transparent conductors need to achieve superior optical transparency, electrical conductivity and mechanical flexibility simultaneously. The length and the diameter of the Ag NWs play very important roles in conductive metal NW network electrodes for flexible transparent conductor. However, the most of metal NWs synthesized by wet chemistry were limited to 1-20 $\mu \mathrm{m}$ in length and showed limited transparency, electrical conductivity and mechanical robustness. This paper reports a method for ultra-long AgNWs with just one step route and apply them as an electrode to Si/PEDOT: PSS solar cells. Through the only one step route, we could dramatically increase the length of the AgNWs form $10 \mu \mathrm{m}$ to even over $100 \mu \mathrm{m}$ with the development of a novel intermittent growth method. By intermittently adding Ag+ solution, the length of nanowires could be controlled in the range of 20-100 $\mu \mathrm{m}$ while diameter of $100 \mathrm{~nm}$ with narrow size dispersion was maintained. This may be because AgNWs to grow along the original nanowire when we add silver again. In addition, Si nanoarray (SiNA)/PEDOT: PSS hybrid solar cells are fabricated with AgNWs electrodes. And the efficiency of solar cells with ultra-long AgNWs electrodes can reach $6 \%$ which is $40 \%$ higher than that of ITO electrodes. The ultra-long AgNWs can be applied for various opto-electronics and ultimately for future wearable electronics.

\section{Acknowledgements}

This work was financially supported by the Hubei Natural Science Foundation (2016CFB266), and State Key Laboratory of Materials Processing and Die \& Mould Technology, Huazhong University of Science and Technology (P2018-012), and Science and technology research project of Hubei Provincial Education Department (B2016442), and Doctoral independent research talent fund (2015CYBSKY005).

\section{References}

[1] Xin Y., Chen Z. and Wang J., et al.: Nanoscale Res. Lett., Vol. 12 (2017), p. 14.

[2] An J. S., Hong S. K. and Kwon O. K.: IEEE T. on Consum. Elect. Vol. 62 (2017), p. 341-348.

[3] Jennings L., Ssewamala F. M. and Nabunya P.: Aids Care, Vol. 28 (2017), p. 273.

[4] Salmon P. M., Goode N. and Taylor N., et al.: Appl. Ergon., c 637-648. 
[5] Guo W. and Liu B.: Acs Appl. Mater. Inter., Vol. 4 (2017), p. 7036-42.

[6] Hecht D. S., Hu L. and Irvin: Adv. Mater., Vol. 23 (2011), p. 1482.

[7] Crespo G. A., Macho S. and Rius F. X.: Anal. Chem., Vol. 80 (2017), p. 1316-22.

[8] Wu C., Yang Y. and Dong D.: Small, Vol. 13 (2017).

[9] Silva L. V. D., Lopes C. B. and Silva W. C. D.: Microchem. J., Vol. 133 (2017), p. 460-467.

[10] Wu D., Lu G., Zhang R., et al.: Electrochim. Acta, Vol. 236 (2017), p. 297-306.

[11] Nechiyil D., Vinayan B. P. and Ramaprabhu S.: J. Colloid Interf. Sci., Vol. 488 (2017), p. 309-316.

[12] Taylor I. M., Robbins E. M. and Catt K. A.: Biosensors Bioelectron., Vol. 89 (2017), p. 400-410.

[13] Diao S., Zhang X. and Shao Z., et al.: Nano Energy, Vol. 31 (2017), p. 359-366.

[14] Oh S., Hong S. and Kim H. J., et al.: Ceram. Int., Vol. 43 (2017), p. 5781-5788.

[15] Long D. X. and Noh Y. Y.: J. of Nanosci. Nanotechno., (2017).

[16] Frisco S., Liu D. and Kumar A., et al.: Acs Appl. Mater. Inter., (2017).

[17] Liu W., Chen L. and Yan J., et al.: Corros. Sci., Vol. 94 (2015), p. 114-121.

[18] Ke F. S., Liu X. C. and Wu J., et al.: Catalysis Today, (2016).

[19] Zhang L., Ding Y. and Li R., et al.: Microchimica Acta, (2017), p.1-7.

[20] Schubert I., Huck C. and Kröber P., et al.: Adv. Optic. Mater., Vol. 4 (2016), p.1838-1845.

[21] Chauvin A., Delacôte C. and Molina-Luna L., et al.: Acs Appl. Mater. Inter., Vol. 8 (2016), p. 6611.

[22] Tyagi N., Jaiswal N. K. and Kovačević G, et al.: Computational Materials Science, Vol. 130 (2017), p. 222-231.

[23] Polívková M., Štrublová V., Hubáček T., et al.: Mat. Sci. Eng. C- Materials, Vol. 72 (2017), p. 512.

[24] Zhang Z., Wu Y., Wang Z., et al.: Mat. Sci. Eng. C, Vol. 78 (2017), p. 706-714.

[25] Kim S., Jeon H. R., An C. H., et al.: Mater. Lett., Vol. 193 (2017), p. 63-66. 